\title{
ON FUNDAMENTAL SOLUTIONS OF ELLIPTIC EQUATIONS ${ }^{1}$
}

\section{AVNER FRIEDMAN}

In this paper we prove: Let $L$ be an elliptic operator in $(x, t)$ with constant coefficients, the coefficients of the lower derivatives being zero, and let $L_{0}$ be the operator obtained from $L$ by deleting all the terms involving $t$-derivatives. Let $K(x, t)$ be a fundamental solution of $L u=0$. Then $\int_{-\infty}^{\infty}[K(x, t)-S(x, t)] d t$ is a fundamental solution of $L_{0} v=0$, where $S(x, t)$ is a certain simple expression introduced for reasons of convergence. [1].

A similar result for parabolic equations was proved by Eidelman

Consider the elliptic equation of order $2 m$

$$
L u \equiv \sum_{k=0}^{2 m} L_{k} D_{t}^{k} u \equiv \sum_{k=0}^{2 m-1}\left(\sum_{|i|=2 m-k} a_{i}^{k} D_{x}^{i} D_{t}^{k} u\right)+D_{t}^{2 m} u=0
$$

where $x=\left(x_{1}, \cdots, x_{n}\right), D_{\lambda}=D_{x_{\lambda}}=\partial / \partial x_{\lambda}(1 \leqq \lambda \leqq n), i=\left(i_{1}, \cdots, i_{n}\right)$, $|i|=i_{1}+\cdots+i_{n}, D_{x}^{i}=D_{1}^{i_{1}} \cdots D_{n}^{i_{n}}$, and where $a_{i}^{\boldsymbol{k}}$ are real constants. Clearly, $L_{0}$ is also elliptic.

A fundamental solution $K(x, t)$ of $L u=0$ is a function defined for $(x, t) \neq 0$ which satisfies the two properties:

(i) $L K=0$ for all $(x, t) \neq 0$,

(ii) for any function $v(x, t)$ in $C^{2^{m}}(\bar{G}), G$ being any bounded domain with boundary $\partial G$ of class $C^{2 m}$,

$$
\begin{aligned}
v(x, t)= & \int_{G} K(x-\xi, t-\tau) L[v(\xi, \tau)] d \xi d \tau \\
& +\int_{\partial G} M[K(x-\xi, t-\tau), v(\xi, \tau)] d S
\end{aligned}
$$

where $(x, t)$ is any point of $G$, and $M\left[w, w^{\prime}\right]$ is an appropriate (not uniquely determined) bilinear form with constant coefficients in $D_{x}^{i} D_{\imath}^{\lambda} w, D_{x}^{j} D_{\imath}^{\mu} w,|i|+\lambda+|j|+\mu=2 m-1$.

In $[2 ; 3]$ it is shown that $K(y)(y=(x, t))$ has the form

$$
K(y)=p(y) \log |y|+|y|^{2 m-n-1} \psi(\zeta) \quad(\zeta=y /|y|)
$$

Received by the editors September 2, 1960.

${ }^{1}$ Prepared under Contract Nonr 710(16) (NR 044 004) between the Office of Naval Research and the University of Minnesota. 
where $\psi(\zeta)$ is analytic in $\zeta$ near $|\zeta|=1, p(y)$ is a polynomial of degree $2 m-n-1$ in $y$ if $2 m \geqq n+1$ and $n+1$ is even, and $p(y) \equiv 0$ otherwise.

Let $B$ be a bounded domain in the $x$-space with boundary $\partial B \in C^{2 m}$ and let $V_{\sigma}$ be the cylinder $\{(x, t) ; x \in B, 0<t<\sigma\}$. It is easily seen that (2) can be applied in the domain $V_{2 t}$. Taking $v(x, t)$ to be a function of $x$ only, i.e., $v(x, t) \equiv u(x)$, we get

$$
\begin{aligned}
u(x)= & \int_{V_{2 t}} K(x-\xi, t-\tau) L_{0} u(\xi) d \xi d \tau \\
& +\int_{S_{2 t}} M_{0}[K(x-\xi, t-\tau), u(\xi)] d \Sigma \\
& +\int_{B_{2 t}} M_{1}[K(x-\xi,-t), u(\xi)] d \xi \\
& -\int_{B} M_{1}[K(x-\xi, t), u(\xi)] d \xi \\
\equiv & \sum_{i=1}^{4} T_{i},
\end{aligned}
$$

where $M_{0}$ is related to $L_{0}$ in the same manner that $M$ is related to $L$, $M_{1}\left[w, w^{\prime}\right]$ is bilinear form in $D_{x}^{i} D_{i}^{\lambda} w, D_{x}^{j} w^{\prime},|i|+\lambda+|j|=2 m-1$, and $S_{2 t}, B_{2 t}$ are the lateral boundary and the upper base of $V_{2 t}$, respectively.

In what follows we denote by $A$ any positive constant independent of $t$. We now let $t \rightarrow \infty$ and consider two cases.

CASE I. $2 m-n+1 \leqq 0$. Using (3) we get

$$
\left|T_{3}\right|=\left|\int_{B_{2 t}} M_{1}[K(x-\xi,-t), u(\xi)] d \xi\right| \leqq \frac{A}{t^{2}} \quad(t \geqq 1) .
$$

A similar estimate holds for $T_{4}$. To evaluate $T_{1}, T_{2}$ we first consider the integral

$$
\int_{0}^{2 t} K(z, t-\tau) d \tau=\int_{-t}^{t} K(z, \lambda) d \lambda \equiv G(z, t) .
$$

Using (3) we have, for any $N>1$,

$$
\left|D_{z}^{i} \int_{1}^{N} K(z, \lambda) d \lambda\right| \leqq A \int_{1}^{\infty} \frac{d \lambda}{\lambda^{n+1-2 m+|i|}} \leqq A \int_{1}^{\infty} \frac{d \lambda}{\lambda^{2}} .
$$

Similarly for $\int_{-N}^{-1}$. Hence, as $t \rightarrow \infty, G(z, t)$ converges together with all its $z$-derivatives (and, clearly, also with its mixed $(z, t)$-derivatives) 
to a function $G(z)$ and all its derivatives, provided $z \neq 0$. A bound on $G(z)$ as $|z| \rightarrow 0$ is easily obtained:

$$
|G(z)| \leqq A+\int_{-1}^{1}|K(z, \lambda)| d \lambda \leqq A|z|^{2 m-n} .
$$

Using these remarks and taking $t \rightarrow \infty$ in (4), we obtain

$$
u(x)=\int_{B} G(x-\xi) L_{0} u(\xi) d \xi+\int_{\partial B} M_{0}[G(x-\xi), u(\xi)] d \sigma
$$

and

$$
G(z)=\int_{-\infty}^{\infty} K(z, t) d t
$$

Thus, $G$ satisfies the second property of fundamental solutions. It remains to prove that $L_{0} G=0, z \neq 0$. Recalling that $L K=0$ we find

$$
\begin{aligned}
L_{0} G(z, t) & =\int_{-t}^{t} L_{0} K(z, \lambda) d \lambda \\
& =-\int_{-t}^{t}\left(\sum_{k=1}^{2 m} L_{k} D_{\lambda}^{k}\right) K(z, \lambda) d \lambda \\
& =-\sum_{k=1}^{2 m}\left[L_{k} D_{t}^{k-1} K(z, t)-L_{k} D_{t}^{k-1} K(z,-t)\right]=O\left(t^{-n}\right) .
\end{aligned}
$$

Taking $t \rightarrow \infty$ we obtain $L_{0} G(z)=0$. We have thus proved that $G(z)$ is a fundamental solution of $L_{0} u=0$.

CASE II. $2 m-n+1>0, n \geqq 1$. In this case the integrals $T_{i}$ need not converge, and we must modify the definition of $G$. One way to do it is the following (compare [1]):

Let $a \neq 0$ be an $n$-dimensional point, and consider the polynomial in $x$ with coefficients in $t$ :

$$
S(x, t)=\sum_{|p|=2 m-1} \frac{1}{p !}(x-a)^{p}\left[D_{x}^{p} K(x, t)\right]_{x=a},
$$

where $x^{q}=x_{1}^{q_{1}} \cdots x_{n}^{q_{n}}, q !=q_{1} ! \cdots q_{n} !$. We replace $K(x, t)$ by

$$
K^{*}(x, t)=K(x, t)-S(x, t)
$$

and proceed by the method of Case I.

Using Green's identity with $u(\xi)$ and $K^{*}(x-\xi, t-\tau)$ we obtain, in analogue with (4), 


$$
\begin{aligned}
u(x) & =\int_{V_{2 t}} K^{*}(x-\xi, t-\tau) L_{0} u(\xi) d \xi d \tau \\
& +\int_{S_{2 t}} M_{0}\left[K^{*}(x-\xi, t-\tau), u(\xi)\right] d \Sigma \\
& +\int_{B_{2 t}} M_{1}\left[K^{*}(x-\xi,-t), u(\xi)\right] d \xi-\int_{B} M_{1}\left[K^{*}(x-\xi, t), u(\xi)\right] d \xi \\
& +\int_{V_{2 t}} u(\xi) L S(x-\xi, t-\tau) d \xi d \tau \equiv \sum_{j=1}^{5} T_{j}^{*} .
\end{aligned}
$$

Next, we derive bounds on $K^{*}$ and its derivatives. By Taylor's formula

$$
\left|K^{*}(z, t)\right|=\left|\sum_{|p|=2 m} \frac{1}{p !}(z-a)^{p} D_{z}^{p} K^{*}(\tilde{z}, t)\right| \leqq \frac{A}{t^{n+1}} \quad(t \geqq 1) .
$$

Using differentiability properties of $K$, as follow by (3), we also get, for any $i$,

$$
\left|D^{i} K^{*}(z, t)\right| \leqq \frac{A}{t^{n+1}} \quad(t \geqq 1) .
$$

From these estimates it follows that the integral

$$
G^{*}(z, t) \equiv \int_{-t}^{t} K^{*}(z, \lambda) d \lambda
$$

and all its derivatives tend to

$$
G^{*}(z)=\int_{-\infty}^{\infty} K^{*}(z, \lambda) d \lambda
$$

and all its derivatives, provided $z \neq 0$. We can now proceed, as in Case I, to evaluate the limits of the $T_{j}^{*}$, for $j=1,2,3,4$. As for $T_{5}^{*}$,

$$
\begin{aligned}
T_{5}^{*} & =\int_{B}\left\{\sum_{k=1}^{2 m} \int_{-t}^{t} D_{\lambda}^{k} L_{k} S(x-\xi, \lambda) d \lambda\right\} u(\xi) d \xi \\
& =\int_{B}\left\{\sum_{k=1}^{2 m} D_{t}^{k-1} L_{k} S(x-\xi, t)-D_{t}^{k-1} L_{k} S(x-\xi,-t)\right\} u(\xi) d \xi .
\end{aligned}
$$

From the definition of $S$ it is easily seen that the terms which appear in the last braces are derivatives of $K$ of order at least $2 m-1$. Hence

$$
\left|T_{5}^{*}\right| \leqq \frac{A}{t^{n}}
$$


As already remarked, $T_{3}^{*}, T_{4}^{*}$ are estimated as in Case I. Combining these remarks and taking $t \rightarrow \infty$, we obtain a representation analogous to (6), with $G$ replaced by $G^{*}$.

Having proved that $G^{*}$ satisfies the second property of fundamental solutions of $L_{0} u=0$, it remains to prove that $L_{0} G^{*}(z)=0$ if $z \neq 0$. Noting that $L_{0} S(z, \lambda)=0$, we have

$$
L_{0} G^{*}(z, t)=\int_{-t}^{t} L_{0} K(z, \lambda) d \lambda=\int_{-t}^{t}\left(\sum_{k=1}^{2 m} L_{k} D_{\lambda}^{k}\right) K(z, \lambda) d \lambda=O\left(t^{-n}\right)
$$

as in Case I. Taking $t \rightarrow \infty$ we get $L_{0} G(z)=0$.

We sum up our result in the following theorem.

TheOREM. A ssume that L, given by (1), is an elliptic operator with real constant coefficients and let $K(x, t)$ be a fundamental solution of $L$. If $n \geqq 1$, then a fundamental solution of $L_{0}$ is given by (11), (9), where $S \equiv 0$ if $2 m-n+1 \leqq 0$ and $S$ is given by (8) if $2 m-n+1>0$.

We remark that all the use we made of the formula (3) was in deriving estimates on $K$ and its derivatives. Such estimates can be derived also for equations with variable coefficients. Using such estimates, the theorem can be extended to some classes of elliptic equations with variable coefficients. It can also be generalized to elliptic systems.

\section{REFERENCES}

1. S. D. Eidelman, On the connection between the fundamental matrix-solution of parabolic and elliptic systems, Mat. Sb. vol. 35 (1954) pp. 57-72.

2. F. John, General properties of solutions of linear elliptic partial differential equations, Proceedings of the Symposium on Spectral Theory and Differential Problems, Stillwater, Oklahoma, 1951, pp. 113-175.

3. - Plane waves and spherical means, New York, Interscience Publishers, 1955.

UNIVERSITY OF MiNNESOTA 